\title{
BMJ Vaccination coverage and Open out-of-sequence vaccinations in rural Guinea-Bissau: an observational cohort study
}

\author{
Linda Hornshøj, ${ }^{1}$ Christine Stabell Benn, ${ }^{1,2}$ Manuel Fernandes, ${ }^{1}$ \\ Amabelia Rodrigues, ${ }^{1}$ Peter Aaby, ${ }^{1,2}$ Ane Bærent Fisker ${ }^{1,2}$
}

To cite: Hornshøj L, Benn CS, Fernandes M, et al. Vaccination coverage and out-of-sequence vaccinations in rural Guinea-Bissau: an observational cohort study. BMJ Open 2012;2:e001509. doi:10.1136/bmjopen-2012001509

- Prepublication history for this paper are available online. To view these files please visit the journal online (http://dx.doi.org/10.1136/ bmjopen-2012-001509).

Received 18 May 2012 Accepted 22 October 2012

This final article is available for use under the terms of the Creative Commons Attribution Non-Commercial 2.0 Licence; see http://bmjopen.bmj.com

${ }^{1}$ Bandim Health Project, INDEPTH Network, Bissau, Guinea-Bissau

${ }^{2}$ Research Center for Vitamins and Vaccines (CVIVA), Bandim Health Project, Statens Serum Institut, Copenhagen S, Denmark

Correspondence to Dr Linda Hornshøj; I.hornshoej@gmail.com

\section{ABSTRACT}

Objective: The WHO aims for $90 \%$ coverage of the Expanded Program on Immunization (EPI), which in Guinea-Bissau included BCG vaccine at birth, three doses of diphtheria-tetanus-pertussis vaccine (DTP) and oral polio vaccine (OPV) at 6, 10 and 14 weeks and measles vaccine (MV) at 9 months when this study was conducted. The WHO assesses coverage by 12 months of age. The sequence of vaccines may have an effect on child mortality, but is not considered in official statistics or assessments of programme performance. We assessed vaccination coverage and frequency of out-of-sequence vaccinations by 12 and 24 months of age.

Design: Observational cohort study. Setting and participants: The Bandim Health Project's (BHP) rural Health and Demographic Surveillance site covers 258 randomly selected villages in all regions of Guinea-Bissau. Villages are visited biannually and vaccination cards inspected to ascertain vaccination status. Between 2003 and 2009 vaccination status by 12 months of age was assessed for 5806 children aged 12-23 months; vaccination status by 24 months of age was assessed for 3792 children aged 24-35 months.

Outcome measures: Coverage of EPI vaccinations and frequency of out-of-sequence vaccinations.

Results: Half of 12 -month-old children and $65 \%$ of 24-month-old children had completed all EPI vaccinations. Many children received vaccines out of sequence: by 12 months of age $54 \%$ of BCG-vaccinated children had received DTP with or before BCG and $28 \%$ of measles-vaccinated children had received DTP with or after MV. By 24 months of age the proportion of out-of-sequence vaccinations was $58 \%$ and $35 \%$, respectively, for BCG and MV.

Conclusions: In rural Guinea-Bissau vaccination coverage by 12 months of age was low, but continued to increase beyond 12 months of age. More than half of all children received vaccinations out of sequence. This highlights the need to improve vaccination services.

\section{ARTICLE SUMMARY}

Article focus

- The WHO aims for a $90 \%$ coverage of the Expanded Program on Immunization (EPI) by 12 months of age.

- The third dose of diphtheria-tetanus-pertussis vaccine (DTP3) is used as a proxy for vaccination coverage and performance.

- Several studies indicate that the sequence of vaccinations affects child mortality, but current policies do not take out-of-sequence vaccinations into consideration.

\section{Key messages}

- Only half of all children were fully vaccinated by 12 months of age, but the coverage continued to increase beyond 12 months of age.

- Limiting vaccination services to children less than 12 months of age will lower the effective vaccination coverage considerably.

- More than half of the children received vaccinations out of sequence.

Strengths and limitations of this study

- The study population was sizeable and the continued demographic surveillance has many advantages compared with Expanded Program on Immunization (EPI) household surveys.

- Although the study was representative for rural Guinea-Bissau, it may not necessarily be representative of other low-income settings.

\section{INTRODUCTION}

In 1974, the WHO launched the Expanded Program on Immunization (EPI), to provide routine vaccinations against tuberculosis, polio, diphtheria, tetanus, pertussis and measles to all children. At the time less than $5 \%$ of children worldwide were vaccinated against these diseases. ${ }^{1}$ In most low-income countries the core vaccines in the EPI are BCG and oral polio vaccine (OPV) at birth, diphtheria- tetanus-pertussis vaccine (DTP) 
and OPV at 6, 10 and 14 weeks and measles vaccine (MV) at 9 months of age though new vaccines are now being added in several places. ${ }^{2}$

The Global Immunization Vision and Strategy 20062015 (GIVS) developed by the WHO/UNICEF focused on ensuring equity in access to vaccination and aimed for a $90 \%$ national vaccination coverage and an $80 \%$ vaccination coverage in every district by $2010 .^{3}$

WHO and UNICEF estimates of vaccination coverage are based on reports of vaccinations performed by service providers (administrative data) and surveys of vaccination history (coverage surveys). The estimates refer to vaccinations given during routine immunisation services to children below 12 months of age, not including supplemental activities such as campaigns with OPV and MV. The administrative data are based on the number of vaccine doses administered to the estimated target population, most commonly the estimated number of children surviving their first year of life. ${ }^{4}$ The WHO EPI cluster methodology is commonly used for coverage surveys. Survey results typically report on annual cohorts of children so that all children included have had 12 months to receive all vaccinations, that is, being between 12 and 23 months of age at the time of the survey as shown in figure $1 .^{45}$

Studies from Guinea-Bissau and other low-income countries suggest that the routine vaccinations have effects on mortality beyond that of protecting against the target disease. These effects are referred to as nonspecific effects (NSE). BCG and MV have beneficial effects in lowering overall child morbidity and mortality, ${ }^{6-18}$ whereas DTP may be associated with increased morbidity and mortality. $.^{7-9} 111217-21$ The NSEs depend on the most recent vaccine and giving vaccines out of sequence may therefore affect child mortality. Worryingly several studies have shown that DTP given together with MV or after MV is associated with increased child morbidity and mortality. ${ }^{9-13} 19$ 21-23

The purpose of this study was to determine the overall vaccination coverage of EPI routine vaccinations and to determine the prevalence of out-of-sequence vaccinations in rural Guinea-Bissau. Both outcomes were determined among 12-month-old and 24-month-old children.

\section{METHODS}

\section{Setting}

In 1978, the Bandim Health Project (BHP) was established as a Health and Demographic Surveillance
System (HDSS) in Guinea-Bissau. Outside the capital, Guinea-Bissau is divided into 10 health regions. Since 1990,5 of these regions have been visited biannually by mobile teams from the BHP. At each visit the teams collect information about health, socioeconomic and vaccination status. In 2006, the remaining regions were included in the surveillance and the mobile teams now cover all 10 regions. The study population in the HDSS is based on 182 randomly selected clusters of 100 women of fertile age and their children below 5 years of age living in a total of 258 villages. In the cluster areas all new households are registered and in all households new women and children taking up residence are added. The HDSS cohort is therefore continuously growing.

\section{Study population}

The study population for the present study was extracted from the HDSS cohort. The population base for the 12-month-old children was children born from the 1 January 2004 until 31 December 2006, registered before 12 months of age and resident in the study area when completing 12 months of age. For the 24-month-old children, children born from the 1 January 2004 until 31 December 2005, registered before 24 months of age and resident in the study area when completing 24 months of age were included.

\section{Vaccinations}

Guinea-Bissau follows the standard EPI. Children in the present study were to be vaccinated in accordance with the EPI schedule shown in figure 1, which at the time included only BCG, OPV0-3, DTP1-3 and MV. The booster MV (MV2) is not part of the routine vaccination schedule as the MV coverage in Guinea-Bissau is far from the $80 \%$ criterion for implementation of MV2. ${ }^{24}$ MV campaigns were conducted in 2006 and 2009.

A child in Guinea-Bissau can be vaccinated at hospitals, most health centres and during outreach visits to the villages. All national vaccines are provided through UNICEF and are free of charge for the parents. However, in order for a child to receive its vaccinations it must have a vaccination card. The card is used to register the child's personal information, vaccinations and the weight of the child. Although the card should also be free of charge, it is most often sold by the health workers for the equivalent of $\$ 1$ (unpublished data). Since September 2007, the BHP teams have been

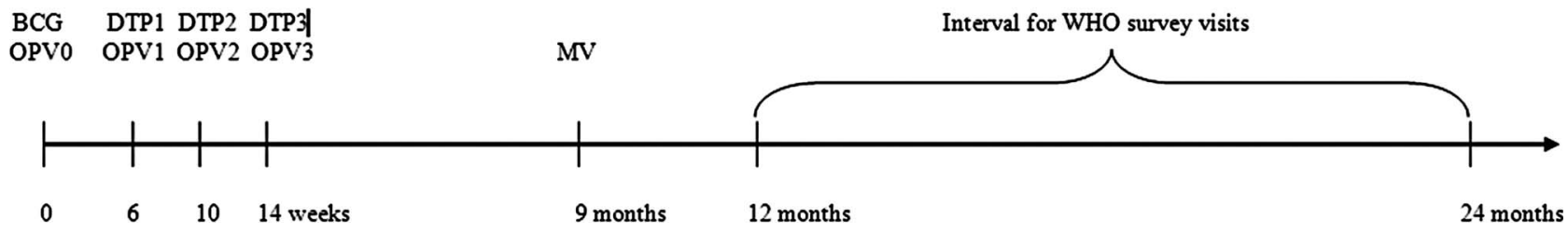

Figure 1 The Expanded Program on Immunization (EPI) schedule for Guinea-Bissau and the age interval for WHO survey visits. DTP, diphtheria-tetanus-pertussis vaccine; MV, measles vaccine; OPV, oral polio vaccine. 
accompanied by nurses who vaccinate children missing doses of OPV, DTP or MV and children receive a vaccination card free of charge. Children were only included in the two cohorts for this study if they had reached 12 or 24 months of age before the BHP nurses began administering vaccines.

\section{Assessment of vaccination coverage}

To emulate the EPI survey methodology used by WHO, we determined the vaccination coverage by 12 months of age in children aged 12-23 months at the time of a home visit. Vaccination status was determined based on the first visit among children aged 12-23 months in 2005-2009 at which vaccination status could be assessed by inspecting the vaccination card. If the child did not have a vaccination card at the time of our visits, it was defined as unvaccinated if the mother confirmed that the child had never received any vaccines. If we were not able to verify a child's vaccinations at any visit in the interval of 12-23 months of age either by (1) seeing the vaccination card or (2) ascertaining that the child had never received vaccinations, the child was not included in the analysis. The vaccination status by 24 months of age was determined using the same method for children visited at 24-35 months of age.

\section{Definitions}

We defined fully vaccinated as completion of the EPI vaccinations not including OPV at birth (OPV0), because very few children receive OPV0 in the rural areas. Out-of-sequence vaccinations were (1) BCG administered together with any dose of DTP, (2) any dose of DTP before BCG, (3) MV with any dose of DTP and (4) any dose of DTP after MV.

\section{Statistical analysis}

Relative risks and 95\% CI were calculated using Poisson regression with robust variance estimates. ${ }^{25}$ Regional

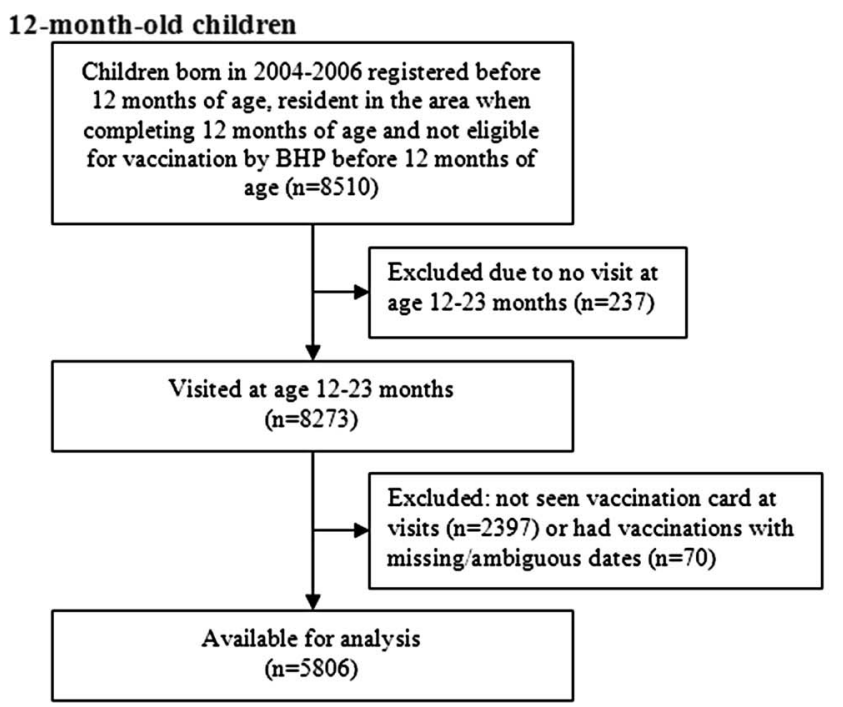

coverages were compared using $\chi^{2}$ tests. Data were analysed using STATA V.11.0.

\section{RESULTS}

We determined vaccination status for 5806 children by 12 months of age and for 3792 children by 24 months of age (figure 2). A larger proportion of children were assessed by 12 months of age $(68 \%)$ than by 24 months of age (59\%), RR=1.15 (95\% CI 1.12 to 1.18$)$.

\section{Vaccination coverage}

By 12 months of age only $50 \%$ of children were fully vaccinated if reception of OPV0 was disregarded. If OPV0 was included in the definition of 'fully vaccinated' only $9 \%$ of 12-month-old children were fully vaccinated. There were large regional differences: only $26 \%$ of children were fully vaccinated in the region of Tombali compared with $65 \%$ in Gabu and Bafata, the regions with highest coverage (table 1). By 24 months of age the percentage of fully vaccinated children was $65 \%$, a $29 \%$ (95\% CI $25 \%$ to $34 \%$ ) increase. The increase ranged from $20 \%$ to $87 \%$ in the different regions (table 1).

Vaccine-specific coverages by 12 months of age were BCG $89 \%$, OPV0 13\%, OPV1 91\%, OPV2 82\%, OPV3 66\%, DTP1 92\%, DTP2 83\%, DTP3 68\% and MV 61\%, with large regional differences (table 2). The drop-out rate between DTP1 and DTP3 was $24 \%$ and the dropout rate between BCG and MV was $31 \%$. There was only a slight increase in the vaccines the children receive early in life when comparing vaccine coverage at 12 and 24 months of age, but the increase for vaccines given later in life was much higher. BCG, OPV1 and DTP1 increased by $3 \%$, whereas OPV3 increased by $12 \%$, DTP3 by $11 \%$ and MV increased by $28 \%$ (figure 3). By 24 months of age, the drop-out rate between DTP1 and DTP3 was $20 \%$ and $13 \%$ between BCG and MV. The DTP3 coverage was higher than the MV coverage by

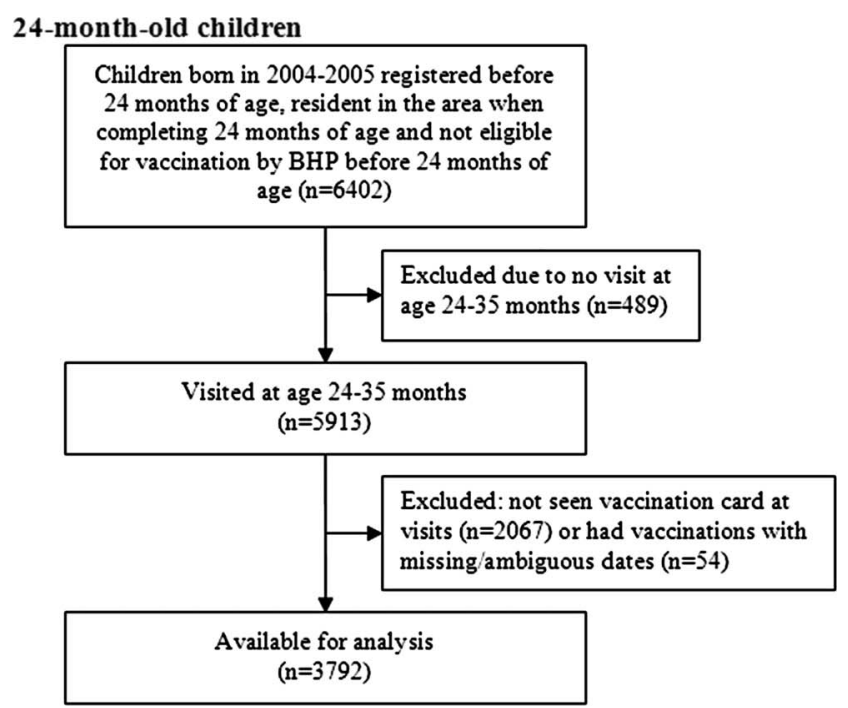

Figure 2 Flow chart of inclusion. BHP, Bandim Health Project. 
Table 1 Vaccination coverage for 12-month-old and 24-month-old children in rural Guinea-Bissau

\begin{tabular}{|c|c|c|c|c|}
\hline \multirow[b]{3}{*}{ Region } & \multicolumn{4}{|c|}{ Vaccination coverage } \\
\hline & \multicolumn{2}{|c|}{$\begin{array}{l}\text { 12-Month-old } \\
\text { children }\end{array}$} & \multicolumn{2}{|c|}{$\begin{array}{l}\text { 24-Month-old } \\
\text { children }\end{array}$} \\
\hline & $\mathbf{n}$ & $\begin{array}{l}\text { Fully } \\
\text { vaccinated } \\
\text { excluding } \\
\text { OPV0 (n (\%)) }\end{array}$ & $\mathbf{n}$ & $\begin{array}{l}\text { Fully } \\
\text { vaccinated } \\
\text { excluding } \\
\text { OPVO (n (\%)) }\end{array}$ \\
\hline All & 5806 & $2903(50)$ & 2446 & $3792(65)$ \\
\hline Oio & 908 & 261 (29) & 212 & $546(39)$ \\
\hline Biombo & 898 & $406(45)$ & 261 & $482(54)$ \\
\hline Gabu & 1071 & $695(65)$ & 510 & $614(83)$ \\
\hline Cacheu & 457 & $265(58)$ & 322 & $224(70)$ \\
\hline Bafata & 855 & $552(65)$ & 492 & $422(86)$ \\
\hline Quinara & 493 & $219(44)$ & 431 & $252(58)$ \\
\hline Tombali & 440 & $115(26)$ & 365 & $178(49)$ \\
\hline Bijagós & 142 & $88(62)$ & 113 & $96(85)$ \\
\hline Bolama & 124 & $75(60)$ & 94 & $75(79)$ \\
\hline $\begin{array}{l}\text { São } \\
\text { Domingos }\end{array}$ & 418 & $227(54)$ & 333 & $217(65)$ \\
\hline $\begin{array}{l}p \text { Value for } \\
\text { same } \\
\text { coverage in } \\
\text { all regions }\end{array}$ & & $<0.0001$ & & $<0.0001$ \\
\hline
\end{tabular}

12 months of age in all regions, except for Tombali. By 24 months of age the overall MV coverage was higher than DTP3, but in four out of 10 regions (Cacheu, Quinara, Bijagós and Bolama) the MV coverage was still lower than the DTP3 coverage (data not shown).

\section{Out-of-sequence vaccinations}

Among children who had received BCG by 12 months of age, $49 \%$ received BCG together with a dose of DTP and $54 \%$ DTP with or before BCG. By 24 months of age these proportions were $52 \%$ and $58 \%$ (table 3 ). The region of
Bijagós managed to stay well below $30 \%$. In comparison in Tombali more than $74 \%$ of children in both age groups received DTP with or before BCG (table 3).

Among children who had received MV by 12 months of age, $26 \%$ received it together with a dose of DTP; this figure was $29 \%$ by 24 months of age. Among the children who had received MV, $28 \%$ had received DTP with or after MV by 12 months of age, and this increased to $35 \%$ by 24 months of age, a $25 \%$ (95\% CI $16 \%$ to $34 \%$ ) increase (table 3). Again there were large regional differences.

\section{DISCUSSION}

\section{Main results}

There were two main observations. First, only half of all children were fully vaccinated by 12 months of age and $65 \%$ by 24 months of age (table 1). Estimating vaccination coverage only by 12 months of age therefore underestimates the final coverage considerably. Many children had initiated the vaccination programme, but not received all the EPI vaccines by 12 months of age. This was reflected in high coverage of vaccines scheduled early in life, with little increase from 12 to 24 months of age, whereas the increase in vaccines scheduled later in infancy was much higher: MV increased by $28 \%$ (25-31\%) (figure 3 ).

Second, a substantial number of children received out-of-sequence vaccinations. More than half of all BCG-vaccinated children received DTP with or before BCG and around a third of MV-vaccinated children received DTP with or after MV (table 3). As vaccination coverage increased among 24-month-old children, so did the frequency of out-of-sequence vaccinations (tables 1 and 3).

\section{Strengths and weaknesses}

The HDSS clusters are randomly selected in all regions. The sample size is substantial and the data obtained are therefore representative of the population in

Table 2 Vaccine coverage among 12-month-old children in rural Guinea-Bissau

\begin{tabular}{|c|c|c|c|c|c|c|c|c|c|c|}
\hline \multirow[b]{2}{*}{ Region } & \multirow[b]{2}{*}{$\mathbf{n}$} & \multicolumn{9}{|c|}{ Vaccine (n (\%)) } \\
\hline & & $\overline{B C G}$ & OPVO & OPV1 & OPV2 & OPV3 & DTP1 & DTP2 & DTP3 & MV \\
\hline All & 5806 & $5146(89)$ & $781(13)$ & $5311(91)$ & 4756 (82) & $3836(66)$ & $5338(92)$ & $4806(83)$ & 3968 (68) & $3567(61)$ \\
\hline Oio & 908 & 740 (82) & $39(4)$ & 787 (87) & $623(69)$ & 393 (43) & 798 (88) & $669(74)$ & $483(53)$ & 445 (49) \\
\hline Biombo & 898 & 765 (85) & $202(22)$ & 793 (88) & $696(78)$ & $554(62)$ & 801 (89) & $701(78)$ & $558(62)$ & $494(55)$ \\
\hline Gabu & 1071 & 999 (93) & 136 (13) & 1020 (95) & $984(92)$ & $870(81)$ & $1023(96)$ & 987 (92) & $882(82)$ & $792(74)$ \\
\hline Cacheu & 457 & 425 (93) & $92(20)$ & $440(96)$ & $412(90)$ & $345(75)$ & 442 (97) & 415 (91) & 359 (79) & $314(69)$ \\
\hline Bafata & 855 & $803(94)$ & $97(11)$ & 816 (95) & 783 (92) & $689(81)$ & $818(96)$ & 785 (92) & 704 (82) & $612(72)$ \\
\hline Quinara & 493 & $422(86)$ & $30(6)$ & $447(91)$ & 387 (79) & 307 (62) & 447 (91) & $385(78)$ & $301(61)$ & $261(53)$ \\
\hline Tombali & 440 & $348(79)$ & $56(13)$ & 357 (81) & $275(63)$ & 160 (36) & 357 (81) & $270(61)$ & $161(37)$ & $204(46)$ \\
\hline Bijagós & 142 & $137(96)$ & 46 (32) & 135 (95) & $132(93)$ & 119 (84) & $135(95)$ & $132(93)$ & $120(85)$ & $95(67)$ \\
\hline Bolama & 124 & 119 (96) & $55(44)$ & $121(98)$ & $118(95)$ & 104 (84) & $121(98)$ & $116(94)$ & $104(84)$ & $80(65)$ \\
\hline São Domingos & 418 & 388 (93) & $28(7)$ & 395 (95) & 346 (83) & $295(71)$ & $396(95)$ & $346(83)$ & $296(71)$ & $270(65)$ \\
\hline $\begin{array}{l}p \text { Value for same } \\
\text { coverage in all } \\
\text { regions }\end{array}$ & & $<0.0001$ & $<0.0001$ & $<0.0001$ & $<0.0001$ & $<0.0001$ & $<0.0001$ & $<0.0001$ & $<0.0001$ & $<0.0001$ \\
\hline
\end{tabular}




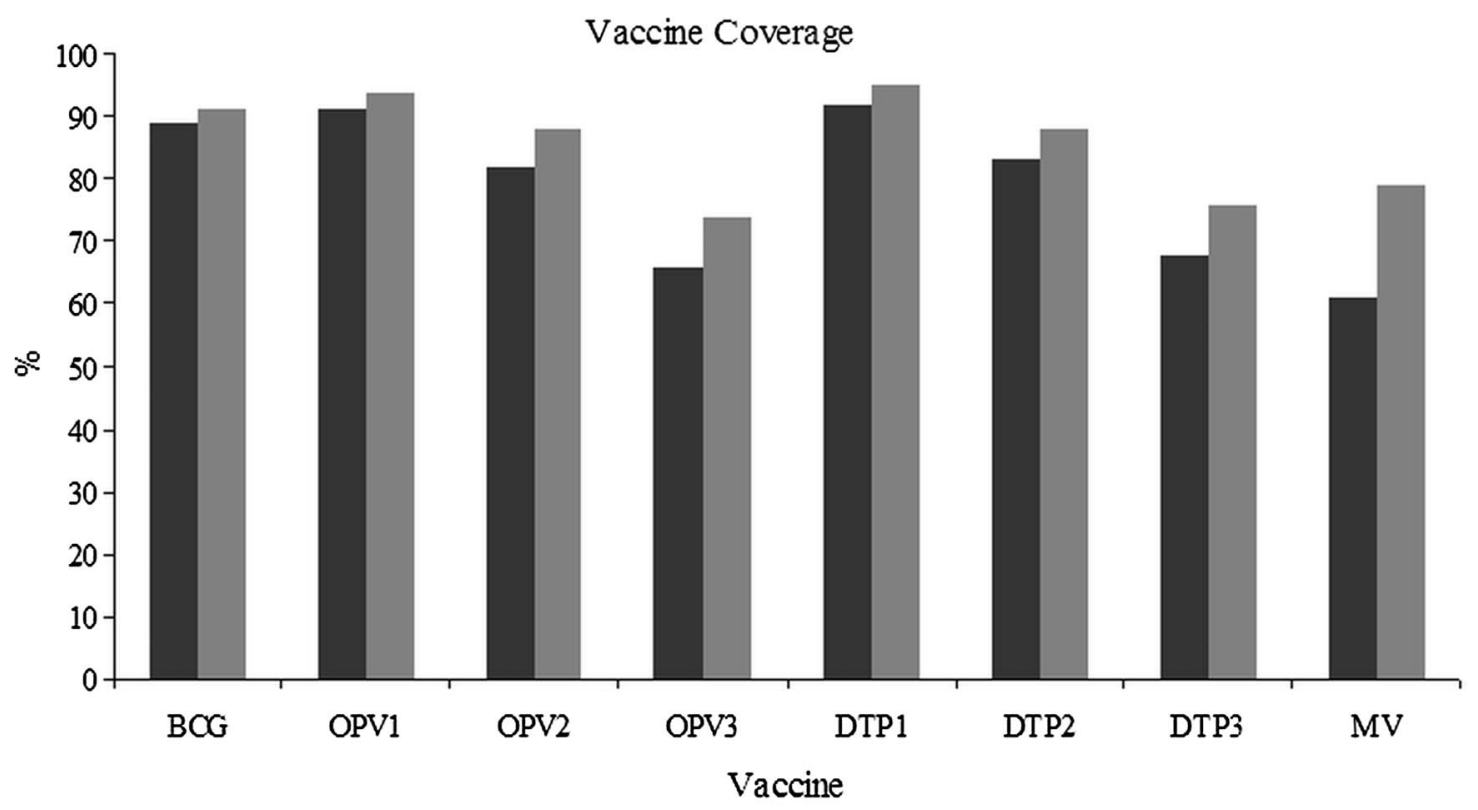

$\square$ By 12 months of age $\square$ By 24 months of age

Figure 3 Vaccine coverage among 12-month-old and 24-month-old children in rural Guinea-Bissau. BCG, bacillus CalmetteGuérin vaccine; DTP, diphtheria-tetanus-pertussis vaccine; MV, measles vaccine; OPV, oral polio vaccine.

Guinea-Bissau excluding the urban area of Bissau. The villages have been visited biannually for years, thus offering the opportunity to verify and correct ambiguous data. We believe that the continued surveillance contributes to more reliable data than other types of surveys. For example, in this study, children with previously registered vaccinations could not be misclassified as unvaccinated at a later visit if the vaccination card had been lost. A study from Malawi showed a BCG scar rate of $70 \%$ among children without documents, making it clear how vaccination coverage can be underestimated if children without vaccination cards are classified as unvaccinated. $^{26}$ We also registered pregnancies and births and could thereby determine date of birth fairly precisely if there was no written record.

\section{Comparison with other studies}

We found that estimating vaccination coverage among 12-month-old children underestimated final coverage. Similarly, Clark and Sanderson found that DTP3 coverage increased from $67 \%$ to $74 \%$ and MV from $59 \%$ to $77 \%$, when comparing the coverage at 12 months of age to the coverage at 36 months of age in survey data from 45 low-income and middle-income countries. ${ }^{27}$

In our study, we emulated the methodology used by the WHO to make the results comparable. A WHO EPI cluster survey was conducted in the capital Bissau in 2005 using vaccination cards or history to confirm vaccination status. It found vaccine coverage to be BCG $87 \%$, OPV3 60\%, DTP3 59\% and MV 71\%, with a $26 \%$ DTP1/DTP3 dropout rate. ${ }^{28}$ When we compared their results with ours, BCG coverage did not differ, but the
WHO OPV3 and DTP3 coverage was 10-13\% (5-18\%) lower and the WHO MV coverage was $16 \%(11-21 \%)$ higher than our estimates (table 2 , ref. ${ }^{28}$ ). The higher MV coverage may in part be explained by our study not including data from the urban Bissau. In Bissau the coverage is higher as vaccinations are more accessible. However, surprisingly higher coverage of all antigens was not found in the WHO survey. If the WHO survey underestimated the true coverage it could in part be due to the use of history, that is, maternal recall, to confirm vaccination status. The validity of recall is correlated to the number of doses received and the age of the child. Mothers tend to recall vaccinations more accurately for younger children with fewer doses and the use of recall seems to underestimate vaccination coverage. ${ }^{29}{ }^{30}$ In the WHO survey, visits are made to randomly selected houses according to the survey method. ${ }^{5}$ Unlike in our study, there are no follow-up visits to households in which the vaccination status for a child cannot be determined at the time of the visit. The WHO EPI survey methodology has previously been criticised for lacking methodological rigour and for introducing bias. ${ }^{31} 32$

\section{Implications}

The vaccination coverage estimates used by WHO and United Nations Children's Fund (UNICEF) emphasises the coverage by 12 months of age, not taking into account the timing of the vaccines received or vaccines received after 12 months of age. ${ }^{33}$ Also, DTP3 is used by funding agencies to evaluate programme performance $^{35}$ and improvements in DTP3 coverage is often linked to additional funding. ${ }^{36}$ This emphasis on 
Table 3 Frequency of out-of-sequence vaccinations among 12-month-old and 24-month-old children in rural Guinea-Bissau

\begin{tabular}{|c|c|c|c|c|c|c|c|c|c|c|c|c|}
\hline \multirow[b]{3}{*}{ Region } & \multicolumn{6}{|c|}{ BCG-vaccinated children } & \multicolumn{6}{|c|}{ MV-vaccinated children } \\
\hline & \multicolumn{3}{|c|}{ 12-Month-old children } & \multicolumn{3}{|c|}{ 24-Month-old children } & \multicolumn{3}{|c|}{ 12-Month-old children } & \multicolumn{3}{|c|}{ 24-Month-old children } \\
\hline & $\mathbf{n}$ & $\begin{array}{l}\text { Received } \\
\text { DTP with } \\
\text { BCG (n (\%)) }\end{array}$ & $\begin{array}{l}\text { Received DTP } \\
\text { with or before } \\
\text { BCG (n (\%)) }\end{array}$ & $\mathbf{n}$ & $\begin{array}{l}\text { Received } \\
\text { DTP with } \\
\text { BCG (n (\%)) }\end{array}$ & $\begin{array}{l}\text { Received DTP } \\
\text { with or before } \\
\text { BCG (n (\%)) }\end{array}$ & $\mathbf{n}$ & $\begin{array}{l}\text { Received } \\
\text { DTP with MV } \\
\text { (n (\%)) }\end{array}$ & $\begin{array}{l}\text { Received DTP } \\
\text { with or after } \\
\text { MV (n (\%)) }\end{array}$ & $\mathbf{n}$ & $\begin{array}{l}\text { Received } \\
\text { DTP with MV } \\
\text { (n (\%)) }\end{array}$ & $\begin{array}{l}\text { Received DTP } \\
\text { with or after } \\
\text { MV (n (\%)) }\end{array}$ \\
\hline All & 5146 & $2520(49)$ & $2770(54)$ & 3455 & $1783(52)$ & $2020(58)$ & 3567 & $924(26)$ & $1012(28)$ & 2981 & $875(29)$ & $1056(35)$ \\
\hline Oio & 740 & $415(56)$ & $466(63)$ & 447 & $256(57)$ & 307 (69) & 445 & $180(40)$ & $196(44)$ & 355 & $163(46)$ & $179(50)$ \\
\hline Biombo & 765 & $364(48)$ & $397(52)$ & 409 & $223(55)$ & $250(61)$ & 494 & $120(24)$ & $133(27)$ & 327 & $110(34)$ & $123(38)$ \\
\hline Gabu & 999 & $526(53)$ & $552(55)$ & 587 & $323(55)$ & 344 (59) & 792 & $201(25)$ & 229 (29) & 559 & $144(26)$ & $178(32)$ \\
\hline Cacheu & 425 & $133(31)$ & $169(40)$ & 305 & $92(30)$ & $125(41)$ & 314 & $43(14)$ & 45 (14) & 259 & 37 (14) & 47 (18) \\
\hline Bafata & 803 & $353(44)$ & $412(51)$ & 477 & $221(46)$ & $258(54)$ & 612 & $128(21)$ & 132 (22) & 460 & $97(21)$ & $106(23)$ \\
\hline Quinara & 422 & $204(48)$ & $214(51)$ & 391 & 193 (49) & 207 (53) & 261 & $66(25)$ & $73(28)$ & 295 & $75(25)$ & 98 (33) \\
\hline Tombali & 348 & $258(74)$ & $263(76)$ & 327 & $252(77)$ & 275 (84) & 204 & $110(54)$ & $124(61)$ & 281 & $159(57)$ & $214(76)$ \\
\hline Bijagós & 137 & $28(20)$ & $39(28)$ & 107 & 24 (22) & 29 (27) & 95 & $7(7)$ & $8(8)$ & 102 & $11(11)$ & 13 (13) \\
\hline Bolama & 119 & 37 (31) & 42 (35) & 85 & $44(52)$ & $52(61)$ & 80 & $9(11)$ & $9(11)$ & 80 & 23 (29) & 25 (31) \\
\hline São Domingos & 388 & 202 (52) & $216(56)$ & 320 & $155(48)$ & $173(54)$ & 270 & $60(22)$ & $63(23)$ & 263 & $56(21)$ & $73(28)$ \\
\hline $\begin{array}{l}p \text { Value for } \\
\text { same } \\
\text { frequency in all } \\
\text { regions }\end{array}$ & & $<0.0001$ & $<0.0001$ & & $<0.0001$ & $<0.0001$ & & $<0.0001$ & $<0.0001$ & & $<0.0001$ & $<0.0001$ \\
\hline
\end{tabular}


vaccinations given during the first year of life and on DTP3 rather than MV may have unwarranted consequences. First, there is less focus on $\mathrm{MV}^{37} 38$ and many African countries now have a lower coverage of MV than DTP3. ${ }^{2}$ Second, the current donor focus on vaccination status by 12 months of age may lead to vaccination providers limiting vaccinations only to children less 12 months of age. This has happened in Guinea-Bissau recently, after the completion of this study. ${ }^{38}$ Our data showed that MV coverage increased by $28 \%$ during the second year of life. Not vaccinating children after 12 months of age provides only a 3-month-window for children to receive routine MV and based on our data it will lead to a considerably lower MV coverage. Added together the emphasis on early vaccinations and on DTP3 rather on MV is likely to lead to more children with DTP as their last vaccination and an increasing number of children not receiving MV. This may be particularly unfortunate as several studies show that MV lowers overall child morbidity and mortality ${ }^{6} 8-14 \quad 16-18$ whereas DTP is associated with increased morbidity and mortality, especially for girls. ${ }^{7-9} 111217-21$

Currently the evaluation of the vaccination programme does not take the frequency of out-of-sequence vaccinations into account. Several studies have indicated that out-of-sequence MV and DTP vaccinations have a negative effect on both child morbidity and mortality. ${ }^{9-13} 19$ 21-23 The frequency of out-of-sequence vaccinations should therefore be included as an indicator of the performance of routine vaccination programmes.

\section{CONCLUSION}

Our study showed that the vaccination coverage in Guinea-Bissau is far from the WHO goal of $90 \%$. Only half of all 12-month-old children were fully vaccinated, with great regional differences in vaccination coverage. Coverage based on vaccinations received during the first 12 months of life significantly underestimated the final coverage. We found that a considerable number of children received out-of-sequence vaccinations. We suggest that vaccination coverage should also be reported by 24 months of age to encourage vaccination of older children and that the proportion of children receiving DTP with or after MV should be the central performance indicator.

The focus on vaccinations to children less than 12 months of age ${ }^{38}$ may lead to a stop of vaccination of older children as experienced recently in Guinea-Bissau. This will obviously restrain many children from completing their vaccination schedule and this negative effect will be the strongest for MV. This will lower herd immunity to measles infections and increase the risk of epidemics. Since all studies show that MV administered as the most recent vaccine has a beneficial effect on child survival ${ }^{6}$ 8-14 $16-18$ it may also have unfortunate consequences that an increasing proportion of children will have DTP as their most recent vaccination. $^{7-9} 111217-21$
Contributors $\mathrm{LH}, \mathrm{ABF}, \mathrm{CSB}$ and PA designed and planned the study. LH, ABF, $M F$ and $A R$ supervised data collection and data entry. LH carried out the statistical analysis with help from ABF, CSB and PA. LH wrote the first draft of the paper and holds the primary responsibility for its final content. All authors contributed to and approved the final manuscript.

Funding This study was supported by the Danish Medical Research Council, the Aase and Ejnar Danielsen's Foundation and DANIDA. The Bandim Health Project (BHP) received support from DANIDA and European Union FP7 support for OPTIMUNISE (grant: Health-F3-2011-261375) to conduct their rural HDSS. CVIVA receives support from the Danish National Research Foundation. CSB is funded by the European Research Council (ERC-2009-StG, grant agreement no. 243149). PA holds a research professorship grant from the Novo Nordic Foundation. Funding agencies had no hand in study design, execution, data analysis, data interpretation or the writing of this paper.

Competing interests None.

Ethical approval No ethical approval has been sought for this study as its data is observational and derived from the BHP's HDSS which has been in place in Guinea-Bissau since 1978 and is conducted by request from the Guinean Ministry of Health.

Provenance and peer review Not commissioned; externally peer reviewed. Data sharing statement No additional data are available.

\section{REFERENCES}

1. UNICEF, WHO. Expanding Immunization Coverage. 2011. http:// www.unicef.org/immunization/index_coverage.html. (Accessed 20 Apr 2012)

2. WHO, UNICEF. Immunization summary-a statistical reference containing data through 2010. Geneva, WHO, 2012.

3. WHO, UNICEF. GIVS: global immunization vision and strategy: 2006-2015. Geneva, WHO, 2005.

4. WHO. WHO vaccine-preventable diseases: monitoring system: 2010 global summary. Geneva, WHO, 2010.

5. WHO. Immunization coverage cluster survey: Reference manual. Geneva, WHO, 2005.

6. Aaby P, Samb B, Simondon F, et al. Non-specific beneficial effect of measles immunisation: analysis of mortality studies from developing countries. BMJ 1995;311:481-5.

7. Aaby $\mathrm{P}$, Jensen $\mathrm{H}$, Gomes $\mathrm{J}$, et al. The introduction of diphtheria-tetanus-pertussis vaccine and child mortality in rural Guinea-Bissau: an observational study. Int $J$ Epidemiol 2004;33:374-80.

8. Aaby $P$, Jensen $H$, Rodrigues $A$, et al. Divergent female-male mortality ratios associated with different routine vaccinations among female-male twin pairs. Int J Epidemiol 2004;33:367-73.

9. Aaby $\mathrm{P}$, Vessari $\mathrm{H}$, Nielsen J, et al. Sex differential effects of routine immunizations and childhood survival in rural Malawi. Pediatr Infect Dis J 2006;25:721-7.

10. Aaby P, Ibrahim SA, Libman MD, et al. The sequence of vaccinations and increased female mortality after high-titre measles vaccine: trials from rural Sudan and Kinshasa. Vaccine 2006;24:2764-71.

11. Aaby $P$, Jensen $H$, Walraven $G$. Age-specific changes in the female-male mortality ratio related to the pattern of vaccinations: an observational study from rural Gambia. Vaccine 2006;24: 4701-8.

12. Aaby $P$, Martins $C$, Bale $C$, et al. Sex differences in the effect of vaccines on the risk of hospitalization due to measles in Guinea-Bissau. Pediatr Infect Dis J 2010;29:324-8.

13. Biai S, Rodrigues A, Nielsen J, et al. Vaccination status and sequence of vaccinations as risk factors for hospitalisation among outpatients in a high mortality country. Vaccine 2011;29: 3662-9.

14. Shann F. Commentary: BCG vaccination halves neonatal mortality. Pediatr Infect Dis J 2012;31:308-9.

15. El-Zein M, Parent ME, Benedetti A, et al. Does BCG vaccination protect against the development of childhood asthma? A systematic review and meta-analysis of epidemiological studies. Int J Epidemiol 2010;39:469-86.

16. Kabir Z, Long J, Reddaiah VP, et al. Non-specific effect of measles vaccination on overall child mortality in an area of rural India with high vaccination coverage: a population-based case-control study. Bull World Health Organ 2003;81:244-50. 
17. Kristensen I, Aaby $\mathrm{P}$, Jensen $\mathrm{H}$. Routine vaccinations and child survival: follow up study in Guinea-Bissau, West Africa. BMJ 2000;321:1435-8.

18. Veirum JE, Sodemann M, Biai S, et al. Routine vaccinations associated with divergent effects on female and male mortality at the paediatric ward in Bissau, Guinea-Bissau. Vaccine 2005;23:1197-204.

19. Aaby P, Benn C, Nielsen J, et al. Testing the hypothesis that diphtheria-tetanus-pertussis vaccine has negative non-specific and sex-differential effects on child survival in high-mortality countries. BMJ Open 2012;2:e000707.

20. Aaby $\mathrm{P}$, Ravn $\mathrm{H}$, Roth $\mathrm{A}$, et al. Early diphtheria-tetanus-pertussis vaccination associated with higher female mortality and no difference in male mortality in a cohort of low birthweight children: an observational study within a randomised trial. Arch Dis Child 2012;97:685-91.

21. Agergaard J, Nante E, Poulstrup G, et al. Diphtheria-tetanus-pertussis vaccine administered simultaneously with measles vaccine is associated with increased morbidity and poor growth in girls. A randomised trial from Guinea-Bissau. Vaccine 2011;29:487-500.

22. Aaby $P$, Jensen $H$, Samb $B$, et al. Differences in female-male mortality after high-titre measles vaccine and association with subsequent vaccination with diphtheria-tetanus-pertussis and inactivated poliovirus: reanalysis of West African studies. Lancet 2003;361:2183-8.

23. Aaby P, Biai S, Veirum JE, et al. DTP with or after measles vaccination is associated with increased in-hospital mortality in Guinea-Bissau. Vaccine 2007;25:1265-9.

24. WHO. Weekly epidemiological record. WHO 2009;35:349-60.

25. Barros AJ, Hirakata VN. Alternatives for logistic regression in cross-sectional studies: an empirical comparison of models that directly estimate the prevalence ratio. BMC Med Res Methodol 2003;3:21.
26. Jahn A, Floyd S, Mwinuka V, et al. Ascertainment of childhood vaccination histories in northern Malawi. Trop Med Int Health 2008;13:129-38.

27. Clark A, Sanderson C. Timing of children's vaccinations in 45 low-income and middle-income countries: an analysis of survey data. Lancet 2009;373:1543-9.

28. WHO, UNICEF. Review of national immunization coverage 19802008-Guinea-Bissau. Geneva, WHO, 2009.

29. Suarez L, Simpson DM, Smith DR. Errors and correlates in parental recall of child immunizations: effects on vaccination coverage estimates. Pediatrics 1997;99:E3.

30. Valadez JJ, Weld LH. Maternal recall error of child vaccination status in a developing nation. Am J Public Health 1992;82:120-2.

31. Luman ET, Worku A, Berhane Y, et al. Comparison of two survey methodologies to assess vaccination coverage. Int $J$ Epidemiol 2007;36:633-41.

32. Burton A, Monasch R, Lautenbach B, et al. WHO and UNICEF estimates of national infant immunization coverage: methods and processes. Bull World Health Organ 2009;87:535-41.

33. WHO, UNICEF and World Bank. State of the world's vaccines and immunization. 3rd ed. Geneva, WHO, 2009.

34. WHO. World Health Statistics 2011-indicator compendium. Geneva, WHO, 2011.

35. GAVI alliance. Guidelines on country proposals-for support for: new and underused vaccines. Geneva, GAVI Alliance, 2011.

36. Arevshatian L, Clements $C$, Lwanga $S$, et al. An evaluation of infant immunization in Africa: is a transformation in progress? Bull World Health Organ 2007:85:449-57.

37. Aaby P, Benn CS. Assessment of childhood immunisation coverage. Lancet 2009;373:1428.

38. Fisker A, Hornshøj L, Rodrigues A, et al. The new decade of vaccines: improving DTP-3 coverage and reducing coverage for measles vaccine. An observational study of the introduction of new vaccines in Guinea-Bissau. BMJ 2012. (In review). 